\title{
Attitudes of Canadian researchers toward the return to participants of incidental and targeted genomic findings obtained in a pediatric research setting
}

\author{
Conrad V. Fernandez, MD1, Caron Strahlendorf, $\mathrm{MBBCh}^{2}$, Denise Avard, $\mathrm{PhD}^{3}$, \\ Bartha M. Knoppers, PhD, LLM, LLB ${ }^{3}$, Colleen O'Connell, PhD4, Eric Bouffet, MD ${ }^{5}$, David Malkin, MD ${ }^{5}$, \\ Nada Jabado, MD, PhD ${ }^{6}$, Kym Boycott, MD, PhD and Poul H. Sorensen, MD, PhD ${ }^{8,9}$
}

Purpose: The purpose of this study was to explore the attitudes of genomics researchers in a pediatric setting in the context of regulatory guidance recommending the disclosure of clinically significant research findings.

Methods: A validated 32-item questionnaire was sent to 107 researchers with two large-scale projects (the Canadian Pediatric Cancer Genome Consortium and the Finding of Rare Genes Canada Consortium). We examined researchers' attitudes toward obligations to offer genomic research results (including if the participant was deceased, a relative, or a child), influence of the certainty/severity of the condition on this obligation, and personal experiences.

Results: Of the 107 researchers, 74 (69\%) responded. Researchers did not feel a strong responsibility to look for meaningful incidental results in the research genomic data set $(n=27,37 \%)$. However, once

\section{INTRODUCTION}

Over the past decade, it has been increasingly recognized that a summary of research results should be offered to participants, typically situated in an ethos of respect for the participant. ${ }^{1-4}$ Attention is now being paid to defining the nature of researchers' responsibilities to the sharing of individual ${ }^{5}$ and, in particular, genomic level results..$^{6-10}$

The ethical implications associated with the offer of return of genomic results have exploded in complexity with the enormous depth of data that are generated using various genomicsequencing techniques. ${ }^{11-13}$ Questions that are debated and polarizing include the following: Is there an obligation for researchers to offer individual results? If there is, how does one define what is of sufficient clinical utility to trigger an offer? ${ }^{14}$ How long does such an obligation persist, particularly, if the participant is of pediatric age ${ }^{15}$ What are obligations of researchers to other family members and is that different if the participant is deceased? ${ }^{16}$ Does it make a difference whether the finding is a target of the genomic research or an incidental finding? ${ }^{17,18}$ The availability of detailed guidance related to what, how, to whom, identified, they felt participants had a strong right to receive them, irrespective of being incidental $(n=50,68 \%)$ or primary targets $(n=$ $64,87 \%)$. There was a high degree of support for informing siblings of genomic results $(n=46,62 \%)$, especially for treatable conditions $(n=56,76 \%)$. Less than half of the participants indicated that their research ethics board required an offer of results $(n=34,46 \%)$ or provided a detailed process $(n=16,22 \%)$.

Conclusion: Researchers strongly support the offer of targeted and incidental genomic research results to participants. Greater regulatory guidance is needed for a consistent approach.

Genet Med 2013:15(7):558-564

Key Words: genomics; incidental findings; next-generation sequencing; researchers

and when the results should be disclosed is of particular significance in pediatrics because of the growing autonomy of the child, the will of the parents, and recommendations regarding the reporting of adult-onset conditions in minors.

International and Canadian norms do not provide much guidance in regard these questions. For example, the 2010 Canadian Tri-Council Policy Statement states: "researchers have an obligation to disclose to the participants any material incidental findings discovered in the course of research"19 and expert bodies (e.g., the National Heart Lung Blood Institute) echo this duty. ${ }^{17,20}$ However, the process of how this should be accomplished and the threshold at which this should occur are poorly defined. A recent study demonstrates that at least half of biobanks do not have specific policies regarding the offer of genomic results. ${ }^{21}$ Clearly, there is a potential for wide variation in researchers' practice given the paucity of detailed guidance.

We report the attitudes and experience of researchers with their research ethics boards (REBs) related to the return of genomic results of researchers in two large pan-Canadian consortia exploring rare diseases and high-risk pediatric cancers.

${ }^{1}$ Department of Pediatrics, IWK Health Centre and Dalhousie University, Halifax, Nova Scotia, Canada; ${ }^{2}$ Department of Pediatric Hematology Oncology, BC Children's Hospital and the University of British Columbia, Vancouver, British Columbia, Canada; ${ }^{3}$ Department of Human Genetics, Centre of Genomics and Policy, Faculty of Medicine, Department of Human Genetics, McGill University, Montreal, Quebec, Canada; ${ }^{4}$ Department of Obstetrics and Gynecology, Perinatal Epidemiology Research Unit, IWK Health Centre, Halifax, Nova Scotia, Canada; ${ }^{5}$ Division of Pediatric Hematology/Oncology, Department of Pediatrics, The Hospital for Sick Children, University of Toronto, Toronto, Ontario, Canada; ${ }^{6}$ Department of Pediatrics, McGill University/McGill University Health Center, Montreal, Quebec, Canada; ${ }^{7}$ Department of Genetics, Children's Hospital of Eastern Ontario and the University of Ottawa, Ottawa, Ontario, Canada; ${ }^{8}$ Department of Molecular Oncology, BC Cancer Research Centre, Vancouver, British Columbia, Canada; ${ }^{9}$ Department of Pathology, University of British Columbia, Vancouver, British Columbia, Canada. Correspondence: Conrad Fernandez (conrad.fernandez@iwk.nshealth.ca) 
Understanding the attitudes of genomics researchers, as key participants in the return of results, is an essential step to the development of policy guidance that allows all researchers to meet their obligations in a consistent manner attentive to the potential challenges that exist in this emerging field. Furthermore, although we surveyed researchers in Canadian consortia, the issues raised here are being debated internationally; thus, these findings may be used as part of the many elements considered during policy development in non-Canadian jurisdictions.

\section{MATERIALS AND METHODS}

The study protocol was approved by the IWK Health Centre REB, Halifax, Nova Scotia, Canada. Participants were informed that consent would be implied by return of the questionnaire. No written consent was required by the IWK REB.

\section{Setting}

All researchers participated in one of two consortia funded by Genome Canada and the Canadian Institutes of Health Research Advancing Technology Innovation Through Discovery program (2010 to the present). ${ }^{22}$ The Canadian Pediatric Cancer Genome Consortium (CPCGC) ${ }^{23}$ has as its main objective the discovery of genomic information in high-risk, poor-prognosis pediatric cancers (osteosarcoma, high-grade glioma, diffuse intrinsic pontine glioma, metastatic medulloblastoma, and atypical teratoid rhabdoid tumors) to gain insight into mechanisms of disease and to identify targets for therapy. The study used anonymized samples or archived samples obtained without consent for return of results, and thus there were no plans to offer results to participants in the CPCGC. The Finding of Rare Genes Canada Consortium (FORGE) ${ }^{24}$ has as its main objective the identification of the genetic causes of rare childhood-onset diseases in individuals and families. Affected individuals (of pediatric or adult age) and sometimes unaffected family members were referred to the consortia primarily through medical geneticists. By definition, conditions studied in FORGE are rare, are often autosomal recessive in nature, and often pediatric in onset. Return of relevant individual results facilitated by genetics counselors was offered as part of the signed consent form. Both consortia use next-generation genomic techniques as their main research tool.

\section{Participants}

The survey was distributed to all the 107 researchers identified to be participating in the CPCGC and/or FORGE through master contact lists held at the consortia. Researchers were broadly defined as genomic scientists and those having consortium involvement in clinical recruitment, bioinformatics, and international collaborations. Consent was presumed by the return of the confidential questionnaires. All demographic variables, including defined researchers' role in the consortia, were self-identified.

\section{Study instrument}

The researchers' questionnaire was developed through a review of the literature. In addition, some questions were derived in part and with permission from the Return of Results Survey that was developed by Lynn Dressler and the Ethics and Policy Working Group of the International Cancer Genome Consortium (data not published). The final questionnaire (Supplementary Data online) reflected five key areas, including attitudes regarding researchers' obligations to offer either incidental or target condition genomic research results; influence of the certainty and severity of the identified condition on this obligation; opinions on course of action if participant is deceased, a relative, or a child; personal experiences with return of genomic findings; and research institution's policy and practice. The preannouncement and instructions on the survey asked researchers to focus on their experiences and opinions overall. They were not directly asked of their experience with either the CPCGC or FORGE. Definitions for study-related terms were included on the introductory page (e.g., definitions of incidental finding, rights, analytic validity, and clinical utility). Likert-scale, openand closed-ended questions were constructed by the method of Dillman ${ }^{25}$ and underwent formal content validity rating by genomics researchers $(n=5)$, with a final rating of 0.87 for individual questions and the instrument overall. The Englishlanguage questionnaire had 33 items and pilot testing showed that it took $20-30 \mathrm{~min}$ to complete.

\section{Study design}

A preannouncement was sent 1 week before the questionnaire was distributed by postal mail. The baseline mailing of the selfadministered questionnaires was in March 2012, with a cover letter, incentive gift card, and a stamped return envelope. Two postal mail reminders were sent. In addition, an e-mail memo was sent to the entire cohort at the 5-week mark by the lead investigators of each of the consortia. Completed questionnaires were returned by stamped mailer. All data were entered at the IWK Health Centre in duplicate and inconsistencies were reconciled.

\section{Statistical analysis}

Data were analyzed using SPSS version 14 (IBM, Somers, NY). Descriptive statistics were used to present the majority of the surveys. Missing data and non-response rates were reported as percentages. Pearson's $\chi^{2}$ test, $t$-test, and the Mann-Whitney tests as appropriate were conducted to assess the associations between demographic variables and specific respondent attitudes. All variables for interrogation by univariate analysis were identified a priori. These included self-identified role in the genomics research, years of practice experience, described comfort in discussing genomic results with participants, age and country of most senior training. Multivariate models were planned to generate hypotheses but were not pursued given the univariate findings.

\section{Participants}

\section{RESULTS}

One hundred and nine questionnaires were sent. Two were returned as undeliverable. Of the remaining 107 possible 
Table 1 Demographics of respondents to the CPCGC-FORGE genomics attitudes questionnaire

\begin{tabular}{lc} 
Demographic & Number (\%) \\
\hline Age & $21(28.4 \%)$ \\
$21-40$ years & $40(54.1 \%)$ \\
$41-55$ years & $13(17.6 \%)$ \\
$>55$ years & \\
Consortium affiliation & $60(81.1 \%)$ \\
FORGE & $5(6.8 \%)$ \\
CPCGC & $9(12.2 \%)$ \\
Both & \\
Self-described role in research process & $43(58.1 \%)$ \\
Genomics researcher & $15(20.3 \%)$ \\
Clinician, nongeneticist & $36(51.4 \%)$ \\
Medical geneticist & $7(9.5 \%)$ \\
Other & \\
Years in practice & $2(2.7 \%)$ \\
$<1$ year & $14(18.9 \%)$ \\
$1-5$ years & $18(24.3 \%)$ \\
$6-10$ years & $20(27 \%)$ \\
$11-20$ years & $20(27 \%)$ \\
$>20$ years &
\end{tabular}

Comfort in discussing genetic results with research participants

Very comfortable

$43(58.1 \%)$

Comfortable

$23(31.1 \%)$

Uncomfortable

$6(8.1 \%)$

Very uncomfortable

$1(1.4 \%)$

Country in which the respondent received their most senior training

Canada

$47(63.5 \%)$

USA

$20(27.0 \%)$

Europe

$5(6.8 \%)$

Other

$2(2.7 \%)$

Participants could select more than one answer for some questions; therefore some sections add up to more than $100 \%$.

CPCGC, Canadian Pediatrics Cancer Genome Consortium; FORGE, Finding of Rare Genetic Diseases Consortium.

respondents, 74 (69\%) completed the questionnaire. The missing-data rate was low. Detailed respondent demographics are shown in Table 1.

\section{Attitudes regarding researchers' obligations to offer either} incidental or target condition genomic research results. Respondents did not feel a strong responsibility to look for meaningful incidental results in the genomic data set they created $(n=12,16 \%)$ (Figure 1 Q1). Researchers indicated that if an incidental result was noted, they had an obligation to offer these results $(n=58,78 \%)$, although this was not unanimous (Figure $1 \mathrm{Q} 2$ ). However, respondents said that once potentially significant results were identified, participants had a right to receive validated genomic results in the majority of $(n=25$,

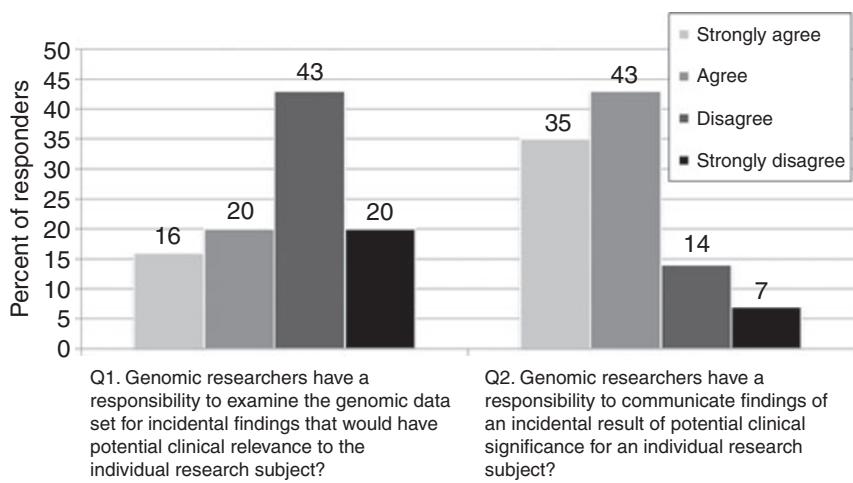

Figure 1 CPCGC and FORGE researcher descriptions of their personal responsibility to offer incidental genomic research results to participants $(\boldsymbol{n}=\mathbf{7 4})$. CPCGC, Canadian Pediatric Cancer Genome Consortium; FORGE, Finding of Rare Genes Canada Consortium.

$34 \%)$ or almost all circumstances $(n=39,53 \%)$ if the findings were related to the primary condition under study. With respect to incidental findings, researchers indicated that most participants had a right to receive results $(n=50,68 \%)$, but just over a quarter said that participants had a right in a minority of circumstances or under no circumstances to receive incidental findings ( $n=22,30 \%)$.

In univariate analysis, we examined the self-reported role as medical geneticist, genomics researcher, or nongeneticist clinician, age, years in practice, country of most senior training, and comfort in discussing genomic results. None of these played a significant factor in reported opinions about a participant's right to target or incidental findings, or subsequent obligations of researchers to return results. Medical geneticists ( $n=15$ of $26,57 \%)$ were significantly more likely than genomics researchers ( $n=6$ of $34,18 \%$ ) to report a feeling of responsibility to examine the data set for incidental clinically relevant findings. $\left(\chi^{2} 10.498, P=0.0001\right)$. We did not have the data to examine whetherMD versus non-MD status explained this difference.

The majority thought that analytic validity should be confirmed using a second sample from the participant $(n=53$, $72 \%$ ), although some felt that the same sample could be used $(n=20,27 \%)$. Respondents were also asked to comment on confirmation strategies for analytic validity (Table 2).

We asked researchers what types of individual genomic research results should be offered to participants (Table 3). A very small minority indicated that raw data should be available to participants (e.g., the DNA sequence or mRNA expression pattern) $(n=8,11 \%)$.

Most indicated that genetic counseling should be offered either almost always or frequently before genetic research participation $(n=48,65 \%)$. This increased substantially $(n=61$, $83 \%$ ) when asked if genetic counseling should be offered before the return of research results, once these were available.

For those who indicated that researchers had a responsibility to offer genomic research results, the duration of this responsibility was primarily linked to the project period $(n=38,51 \%)$ or by ongoing access to a database holding the results $(n=16$, 
Table 2 Strategies indicated by the respondents of the Canadian Pediatrics Cancer Genome Consortium and Finding of Rare Genetic Diseases Consortium as required to confirm analytic validity before the return of genomic research results to participants

The following must be done to confirm analytic validity before return of results to participant n (\%)

Same laboratory that found the result and the same technology

Same laboratory that found the result using an alternative technological strategy

Another research laboratory using the same technology

$30(40.5 \%)$

Another research laboratory using an alternative strategy

$8(10.8 \%)$

A certified clinical laboratory

$13(17.6 \%)$

$51(68.9 \%)$

Other

$5(6.8 \%)$

Answers exceed $100 \%$ because respondents could choose more than one option.

Table 3 Types of individual genomic research results that should be offered to participants as indicated by Canadian Pediatrics Cancer Genome Consortium and Finding of Rare Genetic Diseases Consortium researchers

A validated result should be offered if

The genomic result has clinical utility for the individual

The genomic research result is different from the clinical diagnosis (e.g., genomic result suggests myeloid rather than lymphoblastic leukemia)

The genomic result is available as a clinical test (e.g., BRCA1 53 (71.6\%) mutation)

The genomic result is not available as a clinical test (e.g., new $23(31.1 \%)$ polymorphism associated with response to chemotherapy)

The genomic result is not related to the aims of the research $35(47.3 \%)$ study (e.g., BRCA1 identified during research on diabetes)

I do not think genomic research results should be offered to participants

Answers exceed $100 \%$ as respondents could choose more than one option.

$22 \%)$. However, a few $(n=10,14 \%)$ indicated that responsibility to return results continues indefinitely.

Influence of the certainty and severity of the identified condition on the possible obligation to offer genomic research results. We asked researchers to indicate what threshold measures or factors should be considered to determine whether analytically valid incidental findings should be offered to participants. No one measure was indicated by the majority (Table 4).

There was no consensus among researchers regarding how to resolve and what to do with genomic research results of uncertain clinical significance. A minority said that the decision rested solely with the researcher $(n=9,12 \%)$, whereas others indicated that it should be a joint participant/researchers' decision $(n=18,24 \%)$. Approximately one-third said that results should be returned for the consulting physician or medical geneticist to decide ( $n=27,37 \%)$, whereas a few indicated that an independent committee made up of members of the public
Table 4 Factors that should be used to determine whether analytically valid incidental findings should be offered to participants as indicated by Canadian Pediatrics Cancer Genome Consortium and Finding of Rare Genetic Diseases Consortium respondents

Factors that should be used to determine an offer of results

n (\%)

a. The results should be offered based on exceeding a minimum absolute risk (e.g., >50\% chance for a condition to develop)

b. The results should be offered based on exceeding a minimum relative risk (RR) (e.g., RR of $>3$ for a condition to develop)

c. The results should be offered based on the severity of the condition predicted by the incidental genetic finding (e.g., confers a high risk of death)

d. The results should be offered only if there are variants that meet a list of genes to be determined before commencing the study

e. Incidental findings should not be offered

Other, e.g., if the condition is actionable $(n=8)$

Answers exceed $100 \%$ because respondents could choose more than one option.

and scientists should decide whether results of uncertain significance should be offered to research participants $(n=9,12 \%)$.

Opinions on the course of action if the participant is deceased, or a child, or has a relative potentially impacted in considering the return of genomic results. We asked participants to consider the following scenario. "Sometimes a child or adult with a genetic condition dies before the results of the genetic research testing is available." In the event of death without specific instructions, the vast majority of respondents indicated that the results should be offered to the parents or next of kin, including siblings ( $n=60,81 \%)$. Researchers were then asked to comment on a scenario in which tissue samples for genomic testing had been collected from a child and the child is now $>18$ years of age and cannot be found to confirm consent. In this situation, a slight majority said the tissue should be used only for the original research purposes $(n=$ $44,60 \%)$. Fewer indicated other options, such as allowing for new research purposes $(n=11,15 \%)$, destruction of the tissue $(n=10,14 \%)$, or asking parental consent $(n=17,23 \%)$, were acceptable.

Respondents show a high degree of support for informing siblings of research participants about the risk that they might carry a gene that causes a serious condition, especially if an intervention to ameliorate the condition is feasible $(n=56$; $76 \%$ ). Some researchers said that siblings have few or no rights to be informed about a risk that they might carry a gene that causes a serious condition when no treatment is available. On the other hand, $35 \%$ of these researchers felt that siblings have strong or very strong rights to be informed of a genetic risk discovered in research when treatment is possible $(P=0.004$, $\mathrm{McNemar}$ test). Self-identification as a medical geneticist versus a genomics researcher, age, years in practice, country of most 
senior training, and comfort in discussing genomic results had minimal impact by $\chi^{2}$ analysis on whether researchers endorsed the return of results to siblings, whether actionable or not.

We asked researchers to consider the scenario in which children who participated in genomic research are now adults capable of fully informed consent. We then asked what responsibility, if any, researchers have to ensure that results with potential clinical utility are eventually offered to these participants. No consensus of opinion was noted. A small but significant minority $(n=10,14 \%)$ felt full responsibility to return results extending into adulthood, a few $(n=16,22 \%)$ believed the responsibility should be shared with parents, and almost half believed the responsibility should be shared with a physician $(n=34,46 \%)$. On $\chi^{2}$ analysis, this was not influenced by self-identification as a medical geneticist versus a genomics researcher, age, years in practice, country of most senior training, and comfort in discussing genomic results. In a related question not specific to pediatrics, the majority of researchers indicated that, in general, the duration of any responsibility to return results ends with the project $(n=38,51 \%)$ or access to the database $(n=16,22 \%)$.

The most common free-text comment was that much of the decision about responsibility for return of individual genomic results should be predicated on what is stated in the consent form.

Personal experiences with return of genomic findings. Researchers frequently reported that they encountered genomic information that might be important to the research subject $(n=44,60 \%)$, with some on three or more occasions $(n=27$, $37 \%)$. At least 17 (23\%) had encountered clinically relevant genomic information incidental to the main aims of the study. The majority of researchers reported that they had returned genomic findings at least once to a clinician or research subject $(n=50,68 \%)$.

Policy and practice at respondents' research institutions. Researchers described the frequency with which their REB required an offer to return individual genomic research results to participants as never $(n=16,22 \%)$, sometimes $(n=24,32 \%)$, and always ( $n=10,14 \%)$. Most indicated that either their REB did not have a specific process of how to return individual genomic results $(n=28,38 \%)$ or they were unaware whether one existed $(n=30,41 \%)$. A wide variety of individuals were reported to be involved in the decision to offer individual genomic research results, including genomics researchers $(n$ $=42,57 \%)$, REBs $(n=20,27 \%)$, treating physicians $(n=38$, $51 \%)$, genetic counselors $(n=10,14 \%)$, patients or research subjects $(n=19,26 \%)$, and patient representatives/advocates $(n=6,8 \%)$.

\section{DISCUSSION}

The use of genomic techniques is rapidly uncovering detailed understanding of the genetic mechanisms behind both germline and acquired somatic diseases. ${ }^{26}$ The pace of integration of these techniques has accelerated markedly in the past decade as cost has plummeted and efficiency has risen. ${ }^{27}$ Yet with these advances, ever-evolving ethical challenges confront researchers and practitioners daily across all disciplines. ${ }^{8,9,28}$ Although the Canadian Tri-Council policy statement and other recommendations speak in favor of an obligation to offer individual research results to participants, they typically say little about specific process. Our findings begin to unravel questions raised by this vacuum from the perspective of the genomics researcher.

There are several striking findings in our observations that underline a call for a more robust process description for genomics researchers. ${ }^{10,20}$ In our study, there is no real consensus in what researchers would consider to be thresholds for the return of results. Some would use an elevated relative risk of disease, others an absolute percentage risk, and still others some measure of severity of the condition. Green et al. ${ }^{29}$ describe a high concordance rate for return of a subset of 99 common genetic conditions, but even in this study, specialists in genetic disease did not fully agree for roughly a fifth of these conditions. In addition, in our study, there is no consensus on how hard to look for incidental findings or on how best to ensure analytic validity. We are not aware of any studies that have empirically examined these questions. Various strategies might be considered to avoid the discovery of incidental findings. Examples include anonymization such that tracing data back to the participant is unfeasible, tightly controlled interrogation of limited segments of the genome in predicted areas of interest, or application of filters to exclude known diseasecausing mutations unrelated to the phenotype being explored as has been reported in a neonatal intensive care unit setting. ${ }^{30}$

Of note, the length of obligation to return results is also inconsistently described, some indicating only for the duration of the project and a minority even indefinitely. This latter observation has major resource implications. Most of our respondents agree that once identified, clinically significant results should be offered to participants, their family members and, if deceased, their next of kin. The majority of researchers felt that any ongoing responsibility, presuming one existed, to provide results ended with the closure of the study or access to its database. However, it should be noted that when children were considered, ongoing responsibility alone or with parents or clinicians was frequently described. This underscores that the complex issues in returning genomic results are magnified when the participant is of a pediatric age ${ }^{31}$ and may result in policy that is different for children as compared with adults. Pediatric issues that should be addressed include re-consent when the child reaches the age of majority, duties for providing clinically significant results discovered years after initial enrollment as a child, and duties related to ongoing contact or delegation of responsibility to parents for the purpose of eventually disclosing relevant results. Our findings show no consistent opinion from researchers about how best to approach these challenges.

There are many nuances that should be explored in a qualitative manner to better understand how best to offer results. For example, should results be offered to family members or next 
of kin without the prior consent of either the participant or the family member? How far would such an obligation extend to siblings, parents, children, or even cousins? It is important to recognize that research participants may find utility in data that do not meet scientists' narrower definition of clinical utility. Therefore, should the disclosure of results be limited to findings with personal health implications or expanded to indications that may encompass reproductive planning and other issues with personal meaning? If endorsed, each of these questions would also imply that very significant financial and logistical resources would need to be harnessed to adequately respond to the obligations.

The consortia were responsible to multiple institutional REBs depending on the location of the subproject. Multiple REBs might have influenced the heterogeneity of researchers' responses with respect to their opinions about the return of genomic results, although we think this is less likely because more than threequarters of respondents indicated that their REB did not have a policy or that they were unaware whether one existed. The lack of reported consistent REB guidance means that this approach is likely to be unevenly applied across studies and thus that participants are treated unequally. The lack of review board consistency in biobank research has previously been noted..$^{32}$ Researchers in our report echo those of Meacham in a qualitative study of National Institutes of Health-funded genomics and non-genomics researchers ${ }^{33}$ in which the majority would offer a specific incidental finding related to colorectal cancer to participants.

In considering the appropriate setting for validation of findings before returning them to participants, the variety of opinions is quite striking. Although the majority felt that the finding could be validated in the same laboratory, a significant minority felt it should be in a different laboratory or with a different technology. In the United States, the law prohibits the return of results for clinical use outside of those obtained in a properly certified laboratory (Clinical Laboratory Improvement Amendments (CLIA)). ${ }^{34}$ Having all genomic results retested in a CLIA-certified laboratory poses significant challenges related to the expertise of clinical laboratories, limited samples, and the process by which one retrieves a duplicate sample. There remains a controversy in the literature regarding whether CLIA fully apply to genomic research testing. ${ }^{17}$

We were also surprised that a higher number of researchers did not think that they should return a result that suggests a change in the patient's existing diagnosis. We speculate that researchers did not indicate such a responsibility because of (i) lack of clinical quality assurance (e.g., Clinical Laboratory Improvement Amendments), (ii) elapsed time from sample collection to research study, or (iii) lack of a clinical correlation. A standard guiding all genomics researchers for replication of findings before sharing of the result for clinical use would therefore be extremely useful.

The strengths of these observations are the expert genomics researcher population captured by surveying these two major consortia, the strong pediatric expertise of the participants, the use of a validated questionnaire, and the high response rate. It should also be noted that although these two consortia were constructed to facilitate the genomic discovery goals, many of the researchers worked independently and were geographically widely dispersed; therefore, homogeneity of consortium experience was likely not a strong influence on responses. A limitation of these observations is the potential for respondents reporting socially favorable responses, although we feel this is less likely given the promise of confidentiality for the responses. We also created brief genomic research scenarios for the questionnaire but recognize that these may not precisely mimic the complexities of real-life encounters. A more nuanced approach to some of these very challenging questions, including whether specific cutoff levels for the return of results (as described in Table 3) or a binning system as proposed by Berg, ${ }^{35}$ are helpful to researchers and are likely better addressed in a qualitative-interview format. Particular attention needs to be paid to the small but significant minority who challenge the notion that researchers have any obligation to offer individual results. Exploring their objections in more depth is important to ensure that we are not missing significant pitfalls. Finally, because the researchers in this study represented genomics experts in rare diseases and pediatric cancer, it is possible that researchers in other disciplines or researchers studying healthy participants would view their responsibilities differently. This may be particularly true of non-genomics researchers as the current literature very much focuses on incidental findings emerging from a genomics setting.

This study shows that the genomic research community feels a strong responsibility to offer individual research results to participants and family members, especially if the findings are clinically actionable. A number of respondents indicated that responsibilities should flow from what is stated in the original consent form. However, researchers also identify that REBs often do not have policies regarding the return of genomic results to guide their actions and, when they do, the specific process is often not stipulated. This lack of specific process guidance and the wide variety of opinions on whether and how to act on individual research results mean that it is likely that research participants are very inconsistently treated. We are gathering parallel opinions from participants in genomic research that, together with the current findings, will, we believe, inform the process by which the Canadian Tri-Council Policy Statement can be more uniformly implemented and honor the moral obligations we have to our research participants. These findings are very likely to be informative across many jurisdictions.

\section{SUPPLEMENTARY MATERIAL}

Supplementary material is linked to the online version of the paper at http://www.nature.com/gim

\section{ACKNOWLEDGMENTS}

The researchers thank the expert reviewers for the pilot study in validation of the questionnaire. We also thank Aleasha Warner (research associate, IWK Heath Centre) and Janet Macardier (genetics counselor and research associate, Children's Hospital 
of Eastern Ontario) for their superb efforts on this project. We acknowledge Lynn Dressler and the Ethics and Policy Working Group of the International Cancer Genome Consortium (ICGC) for generously sharing their researcher questionnaire. This questionnaire was presented in abstract form at the $6^{\text {th }}$ Annual Meeting of the ICGC, Cannes, France, March 2012. Lynn Dressler, Janell Markey, Allison Deal, and Bartha Knoppers worked on behalf of the Ethics and Policy Working Group of the ICGC. Bartha Knoppers is funded in part by NHGRI 1R21HG0061201 (Clayton) (Returning research results of pediatric genomic research to participants). Return of individual results from cancer genomic research: ICGC researcher experiences and institutional practices. Funding for this project from the Canadian Pediatric Cancer Genome Consortium (CPCGC) and the Finding of Rare Genes Canada Consortium (FORGE) have both been sponsored by Genome Canada and the Canadian Institutes of Health Research "Advancing Technology Innovation Through Discovery" program and Health Canada. Previous presentations: Data from this article was presented, in part, at the American Society of Hematology Annual Meeting, Atlanta, Georgia, December 2012. The work presented in this article is original and has not been previously published in article form.

\section{DISCLOSURE}

The authors declare no conflict of interest.

\section{REFERENCES}

1. Fernandez $C V$, Weijer $C$. Obligations in offering to disclose genetic research results. Am J Bioeth 2006;6:44-46; author reply W10-42.

2. Partridge AH, Burstein HJ, Gelman RS, Marcom PK, Winer EP. Do patients participating in clinical trials want to know study results? I Natl Cancer Inst 2003:95:491-492.

3. Shalowitz DI, Miller FG. Communicating the results of clinical research to participants: attitudes, practices, and future directions. PLoS Med 2008;5:e91.

4. Rigby H, Fernandez CV. Providing research results to study participants: support versus practice of researchers presenting at the American Society of Hematology annual meeting. Blood 2005;106:1199-1202.

5. Shalowitz DI, Miller FG. Disclosing individual results of clinical research: implications of respect for participants. JAMA 2005;294:737-740.

6. McGuire AL, Caulfield T, Cho MK. Research ethics and the challenge of wholegenome sequencing. Nat Rev Genet 2008;9:152-156.

7. Beskow LM, Burke W. Offering individual genetic research results: context matters. Sci Trans/ Med 2010;2:38cm20.

8. Wolf SM, Lawrenz FP, Nelson CA, et al. Managing incidental findings in human subjects research: analysis and recommendations. J Law Med Ethics 2008;36:219-248, 211

9. Caulfield T, McGuire AL, Cho M, et al. Research ethics recommendations for whole-genome research: consensus statement. PLoS Biol 2008;6:e73.

10. Zawati MH, Knoppers BM. International normative perspectives on the return of individual research results and incidental findings in genomic biobanks. Genet Med 2012;14:484-489.

11. McGuire AL, Basford M, Dressler LG, et al. Ethical and practical challenges of sharing data from genome-wide association studies: the eMERGE Consortium experience. Genome Res 2011;21:1001-1007.

12. Tabor HK, Berkman BE, Hull SC, Bamshad MJ. Genomics really gets personal: how exome and whole genome sequencing challenge the ethical framework of human genetics research. Am J Med Genet A 2011;155A:2916-2924.

13. Guttmacher AE, McGuire AL, Ponder B, Stefánsson K. Personalized genomic information: preparing for the future of genetic medicine. Nat Rev Genet 2010;11:161-165.
14. Bookman EB, Langehorne AA, Eckfeldt JH, et al.; NHLBI Working Group Reporting genetic results in research studies: summary and recommendations of an NHLBI working group. Am J Med Genet A 2006;140:1033-1040.

15. Burke W, Diekema DS. Ethical issues arising from the participation of children in genetic research. J Pediatr 2006;149(1 Suppl):S34-S38.

16. Black L, McClellan KA. Familial communication of research results: a need to know? J Law Med Ethics 2011:39:605-613.

17. Fabsitz RR, McGuire A, Sharp RR, et al. Ethical and practical guidelines for reporting genetic research results to study participants: updated guidelines from a National Heart, Lung, and Blood Institute working group. Circ Cardiovasc Genet 2010;3:574-580.

18. Lockhart NC, Yassin R, Weil CJ, Compton CC. Intersection of biobanking and clinical care: should discrepant diagnoses and pathological findings be returned to research participants? Genet Med 2012;14:417-423.

19. Canadian Institutes of Health Research, Natural Sciences and Engineering Research Council of Canada, and Social Sciences and Humanities Research Council of Canada, Tri-Council Policy Statement: Ethical Conduct for Research Involving Humans. http://www.pre.ethics.gc.ca/pdf/eng/tcps2/TCPS_2_FINAL_ Web.pdf.

20. Wolf $S M$, Crock BN, Van Ness B, et al. Managing incidental findings and research results in genomic research involving biobanks and archived data sets. Genet Med 2012;14:361-384.

21. Johnson G, Lawrenz F, Thao M. An empirical examination of the management of return of individual research results and incidental findings in genomic biobanks. Genet Med 2012;14:444-450.

22. Advancing Technology Innovation through Discovery. http://www. genomecanada.ca/en/portfolio/research/advancing-technology-innovationcompetition.aspx.

23. Canadian Pediatrics Cancer Genome Consortium. http://www.bcgsc.ca/about/ news/the-canadian-pediatric-cancer-genome-consortium. 2012

24. Canadian Pediatric Genetics Sequencing Consortium. http://www. cpgdsconsortium.com. 2012.

25. Dillman D. Mail and Internet Surveys. The Tailored Design Method, 2nd edn. John Wiley \& Sons, Inc.: New York, 2000.

26. Green ED, Guyer MS; National Human Genome Research Institute. Charting a course for genomic medicine from base pairs to bedside. Nature 2011;470:204213.

27. Feero WG, Guttmacher AE, Collins FS. Genomic medicine-an updated primer. N Engl J Med 2010;362:2001-2011.

28. Ruiz-Canela M, Valle-Mansilla JI, Sulmasy DP. Researchers' preferences and attitudes on ethical aspects of genomics research: a comparative study between the USA and Spain. J Med Ethics 2009;35:251-257.

29. Green RC, Berg JS, Berry GT, et al. Exploring concordance and discordance for return of incidental findings from clinical sequencing. Genet Med 2012;14:405410.

30. Saunders CJ, Miller NA, Soden SE, et al. Rapid whole-genome sequencing for genetic disease diagnosis in neonatal intensive care units. Sci Trans/ Med 2012:4:154ra135.

31. Samuël J, Knoppers BM, Avard D. Paediatric biobanks: what makes them so unique? J Paediatr Child Health 2012;48:E1-E3.

32. Wolf LE, Catania JA, Dolcini MM, Pollack LM, Lo B. IRB Chairs' Perspectives on Genomics Research Involving Stored Biological Materials: Ethical Concerns and Proposed Solutions. J Empir Res Hum Res Ethics 2008;3:99-111.

33. Meacham MC, Starks H, Burke W, Edwards K. Researcher perspectives on disclosure of incidental findings in genetic research. J Empir Res Hum Res Ethics 2010;5:31-41.

34. Schwartz MK. Genetic testing and the clinical laboratory improvement amendments of 1988: present and future. Clin Chem 1999;45:739-745.

35. Berg JS, Khoury MJ, Evans JP. Deploying whole genome sequencing in clinical practice and public health: meeting the challenge one bin at a time. Genet Med 2011:13:499-504.

This work is licensed under a Creative Derivative Works 3.0 License. To view a copy of this license, visit http://creativecommons.org/licenses/by-nc-nd/3.0/ 